\title{
Endometrial Carcinoma Presenting with an Isolated Osseous Metastasis: A Case Report
}

\author{
Nesrine TOUNSI ${ }^{1}$, Ines ZEMNI ${ }^{1}$, Fatma SAADALAH ${ }^{1}$, Maher SLIMANE ${ }^{1}$, Nadia BOUJELEBENE ${ }^{2}$, \\ Mohamed Ali AYADI ${ }^{1}$, Riadh CHARGUI ${ }^{1}$, Khaled RAHAL ${ }^{1}$
}

Tunisia

\begin{abstract}
Patients with advanced endometrial cancer had generally a poor prognostic with a median survival often less than one year. Isolated bone metastases as the first sign of the disease of endometrial cancer was a very exceptional situation rarely described in the literature. The goal of this report is to describe the management of this rare site of metastases and to reviewed Clinic pathological features and prognosis of isolated bone metastases. We present a case of a 56-year-old woman treated for endometrial cancer with isolated ribs bone metastases. She was complaining about progressive right rib pain as a first sign of the disease of endometrial cancer. She had a biopsy of the lesion in the last right thoracic rib. This has proved a metastatic adenocarcinoma compatible with an endometrial primary cancer. An endometrial biopsy showed moderately differentiated endometrioid adenocarcinoma. Imagery did not reveal any other sites of metastatic disease. The patient underwent bilateral salpingo-oophorectomy and hysterectomy, peritoneal washing, omentectomy, bilateral pelvic and par aortic lymphadenectomy. In addition, a right thoracotomy was carried out and complete surgical excision of the rib mass was successfully performed. Adjuvant chemotherapy was administrated. The patient is clinically free of disease 10 months following diagnosis. Single-bone extrauterine metastatic site and local disease limited to the uterus had better survival outcomes than Stage IVb endometrial cancer with multiple metastatic sites. Therefore, the patient treated with surgery with a clear margin continued to behave as early-stage endometrial cancer.
\end{abstract}

Keywords: Bone metastasis, Chemotherapy, Endometrial cancer, Isolated metastasis, Rib

Gynecol Obstet Reprod Med 2022;28(1):108-111

\section{Introduction}

Endometrial cancer is the most prevalent gynecologic cancer with a progressively increasing incidence (1). Approximately $(5-10 \%)$ of the patients have been diagnosed with locoregionally advanced stages (III-IVA) disease. ${ }^{2}$ Early

${ }^{1}$ Department of surgical oncology, Salah Azaiz Institute, University Tunis El Manar Tunis, Tunisia

${ }^{2}$ Department of Pathology, Salah Azaiz Institute, University Tunis El Manar Tunis, Tunisia

Address of Correspondence: Tounsi Nesrine

Adresse, Boulevard du 9-Avril 1938

1006 Tunis, Salah Azaiz Institute, Tunisia

neserine.tounsi@gmail.com

SSubmitted for Publication: 18.02.2021 Revised for Publication: 03.05.2021 Accepted for Publication: 17.05.2021 Online Published: 01.06.2021

ORCID IDs of the authors: $\quad$ TN: 0000-0002-8845-4773

IZ:0000-0002-7244-8248 FS: 0000-0003-1976-2112

SM: 0000-0003-0311-847X BN: 0000-0002-4888-8198

MAA: 0000-0001-9294-4348 RC: 0000-0002-4518-4048 KR:0000-0002-3474-0651

\begin{tabular}{c|c}
\hline Quick Response Code: & Access this article online \\
\cline { 2 - 2 } & Website: www.gorm.com.tr \\
& e- mail: info@gorm.com.tr \\
\hline & DOI:10.21613/GORM.2021.1191 \\
\hline
\end{tabular}

How to cite this article: Tounsi N. Zemni I. Saadalah F. Slimane $M$. Boujelebene N. Ayadi MA. Chargui R. Rahal K. Endometrial Carcinoma Presenting with an Isolated Osseous Metastasis: A Case Report. Gynecol Obstet Reprod Med 2022;28(1):108-111 endometrial cancer had a good prognosis contrary to the advanced stage of the disease.

Lymph nodes, liver, and lungs are the most frequent cancer-metastasized sites, while bone metastasis is very rare (3). Moreover, isolated bone metastasis is a very exceptional situation rarely described in the literature $(4,5)$.

The goal of this report is to describe the management of this rare site of metastases and to reviewed Clinic pathological features, treatment, and prognosis of isolated bone metastases.

\section{Case Report}

A 56-year-old post-menopausal, nulliparous woman had been suffering from progressive right rib pain for more than two months without any other associated symptoms. Palpation of the bone mass revealed that it was hard, painful, and adherent to deep structures.

The CT scan of chest imaging revealed a $30 \times 40 \mathrm{~mm}$ mass in the last right thoracic rib measuring 30x40mm expanding into the adjacent soft tissues with bony destruction. The CT scan of the abdomen and pelvic did not reveal other distant metastasis. A bone biopsy of the right ribs lesion was per- 
formed, which proved a moderately differentiated metastatic adenocarcinoma.

Immunohistochemistry performed was positive for Cytokeratin 7 (CK7), 10\% for Ki67, and negative for CK20, and Human epidermal growth factor receptor 2 (HER-2). Additionally, there was $20 \%$ of estrogen receptor (ER) and $70 \%$ of progesterone receptor (PR). Therefore, it was considered as breast or gynecological origin metastatic cancer.

Mammography and echography, as well as tumor markers Cancer antigen 125 (CA125), Carcinoembryonic Antigen (CEA), CA153, and CA199, were normal.

Transvaginal sonography demonstrates an endometrial thickness of $20 \mathrm{~mm}$. The gynecological examination was normal. Magnetic resonance imaging (MRI) describes enlargement of the uterus, with a mass measuring $(26 \times 30 \mathrm{~mm})$ in the endometrial cavity. It was deeply invading the myometrium. The mass showed heterogeneous medium and high signal intensity on T2-weighted images. Moreover, MRI showed a markedly increased uptake mass on the last right thoracic rib measuring 30×40mm (Figure 1).

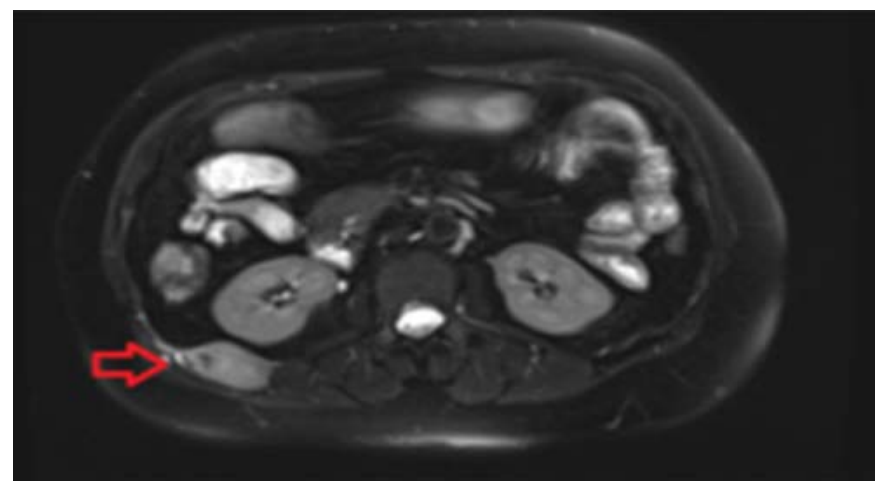

Figure 1: MRI showed a markedly increased uptake mass on the last right thoracic rib measuring $30 \times 40 \mathrm{~mm}$. The mass showed heterogeneous medium and high signal intensity on T2-weighted images.

Hysteroscopy with biopsy confirmed a well-differentiated endometrioid adenocarcinoma grade II. Isotope scan (PET) was not realized because is not available. According to the International Federation of Gynecology and Obstetrics staging system, her disease was classified as stage IVB.

The patient underwent bilateral salpingo-oophorectomy and hysterectomy, peritoneal washing, omentectomy, bilateral pelvic and par aortic lymphadenectomy. In addition, a right thoracotomy was carried out and complete surgical excision of the rib mass was successfully performed.

Histopathology showed primary cancer as a grade 2 endometrial endometrioid adenocarcinoma with more than 50\% myometrial invasion without reaching to serosa. Para-aortic lymphadenectomy sampling revealed one metastatic lymph node. The metastatic lesion of the rib was solid-cystic mea- suring $60 \times 40 \mathrm{~mm}$. The tumor was diffusely infiltrating the bone (Figure 2) with the presence of necrosis and lymphovascular space involvement. Resection was complete with clear margins.

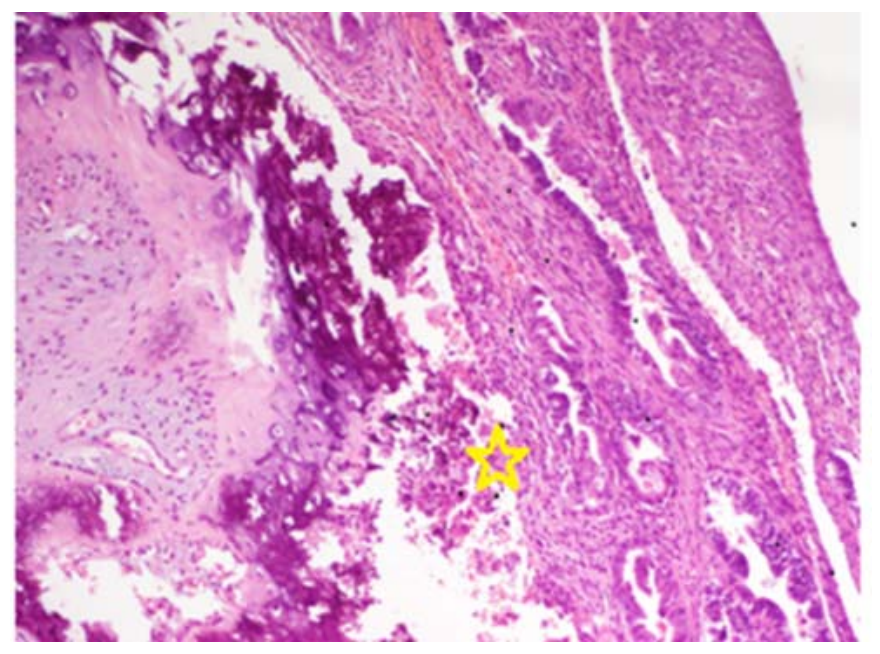

Figure 2: Endometrioid adenocarcinoma infiltrating the bone (HE $\times 100$ ).

The decision of the multidisciplinary team (MDT) was to administrate chemotherapy with paclitaxel and carboplatin.

She is still alive after ten months of treatment without disease progression. Informed consent was obtained from the patient for the publication of the case report.

\section{Discussion}

Bone metastasis in endometrial cancer is exceptional. In literature, their prevalence is variously reported $(0 \%-15 \%)$ $(4,5)$. Most commonly, bone metastases are seen as recurrence. However, their presence as the initial sign of the disease is rare. The vertebrae are the most frequent bone metastatic site, followed by the pelvic bones, femur, ribs, and sternum (6$8)$. Few other sites like bone extremities had rarely described (9). Nevertheless, the mechanisms of dissemination of bone metastases are not fully clear.

Hematogenous spread is considered the most frequent mechanism (10). The literature review, reported by Makris GM et al, revealed 58 case reports with metastatic endometrial cancer to the bone between 1967 and 2016, among them 36 cases were diagnosed with bone metastasis as the initial sign of disease (3). Bone metastasis could present a very interesting diagnostic challenge, often because, gynecological symptoms could occur at a later time (11). Hence, the interest in doing biopsies.

Traditionally, treatment for women with stage IV endometrial cancer relies on palliative chemotherapy. Although, the best therapeutic approach for bone metastases of EC remains unclear (8). Treatment depends on-site, the number of bone lesions, and the presence of concurrent visceral metastases. 
Many authors reveal that the most widely used treatment for metastases to the bone involves surgical resection of the accessible lesion, stereotaxic or palliative radiotherapy, radiofrequency with cementoplasty, systemic chemotherapy or hormonotherapy, and bisphosphonates $(6,10,12)$.

Uharcek et al reported that palliative radiotherapy could be efficient in the case of isolated bone metastasis since it allows the preservation of bone intact with good results $(13,14)$. Moreover, high dose localized radiotherapy followed by chemotherapy can be effective for voluminous tumors. This has been demonstrated by Koukourakis et al, that had reported a patient with peritoneal and pelvic metastasis who had complete remission after stereotactic re-irradiation combined with chemotherapy (15). In the review of the literature including 29 cases, reported by Myriokefalitaki et al, regarding endometrial cancer presenting with bone metastasis as a first symptom, the bone tumor has been treated with radiotherapy alone in $28 \%$ of the cases or combined with primary surgery in $12 \%$ of the cases or chemotherapy in $40 \%$. For the whole, $54 \%$ of patients had chemotherapy and $46 \%$ had hormone therapy (11). In our case, the patient had a complete surgical excision of the rib mass followed by chemotherapy. Therefore, endometrial carcinoma with a solitary bone metastasis needs multimodal therapy.

In the literature, the median OS of patients with bone metastasis was between 18 months and 33 months $(4,5,8)$. Moreover, patients with recurrent bone metastasis have better survival than patients with endometrial carcinoma who developed bone metastasis at presentation $(4,5)$. Furthermore, the prognosis for patients with a single-bone extra uterine metastatic site was better than patients with advanced disease $(3,8,11)$. In addition, patients with Type I endometrial carcinoma who have bone metastasis have a better prognosis comparing to patients with Type II histology (8).

Bone metastasis diagnosed as the first sign of the disease of endometrial cancer are extremely rare. The goal through our case reports and the review of other cases in the literature was to collect as much information exists regarding the best management of patients with stage IV endometrial cancer who developed this rare presentation of the disease. Therefore, we suggest that wide resection with a clear margin of single bone metastasis is the best treatment.

Acknowledgments: We would like to thank Mrs. Ilhem Ben Marzouk for the English Revision.

Funding: This research did not receive any specific grant from funding agencies in the public, commercial, or not-for-profit sectors.

Availability of data and materials: The data supporting this study is available through the corresponding author upon reasonable request.

Declaration of conflicting interests: The author(s) declared no potential conflicts of interest with respect to the research, au- thorship, and/or publication of this article. The authors do not report any financial or personal connections with other persons or organizations, which might negatively affect the content of this publication and/or claim authorship rights to this publication.

Authors' contributions: Acquisition of data: Ines Zemni, Nesrine Tounsi, Nadia Boujelebene; Analysis and interpretation of data: Nesrine Tounsi, Nadia Boujelebene; Critical revision: Ines Zemni, Maher Slimene; Drafting of the manuscript: Mohamed Ali Ayadi, Riadh Chargui, Khaled Rahal; Study conception and design: Nesrine Tounsi, Ines Zemni; Final approval of the version to be published: All authors.

\section{References}

1. Miyahara D, Yotsumoto F, Hirakawa T, Yoshikawa K, Shigekawa K, Miyata K, et al. Clinical features of recurrence in patients without residual tumour in endometrial cancer. Anticancer Res. 2019;39(8):4581-8. Doi: 10.21873/anticanres.13637.

2. Lewin SN, Herzog TJ, Barrena Medel NI, Deutsch I, Burke WM, Sun X, et al. Comparative performance of the 2009 international Federation of gynecology and obstetrics' staging system for uterine corpus cancer. Obstet Gynecol. 2010;116(5):1141-9. Doi: 10.1097/AOG. 0b013e3181f39849.

3. Makris GM, Mene J, Battista MJ, Chrelias G. Sergentanis TN. Psyrri A. et al. Endometrial carcinoma with tibial bone metastasis: a case report and literature review. J Obstet Gynaecol. 2018;38(8):1039-47. Doi: 10.1080/ 01443615.2017.1420759.

4. Kehoe SM, Zivanovic O, Ferguson SE, Barakat RR, Soslow RA. Clinicopathologic features of bone metastases and outcomes in patients with primary endometrial cancer. Gynecol Oncol. 2010;117(2):229-33. Doi: 10.1016/j.ygyno.2010.01.047.

5. Yoon A, Choi CH, Kim TH, Choi JK, Park JY, Lee YY, et al. Bone metastasis in primary endometrial carcinoma: features, outcomes, and predictors. Int J Gynecol Cancer. 2014;24(1):107-12. Doi: 10.1097/IGC. 0000000000000015 .

6. Longo R, Gamelon-Benichou C, Elias-Matta C, Platini C, Eid N, Yacoubi M, et al. An unusual solitary metatarsal metastasis from an endometrioid endometrial adenocarcinoma. Am J Case Rep. 2015;16:473-7. Doi: 10.12659/ AJCR.893978.

7. Cooper JK, Wong FL, Swenerton KD. Endometrial adenocarcinoma presenting as an isolated calcaneal metastasis. A rare entity with good prognosis. Cancer. 1994; 73(11):2779-81. Doi: 10.1002/1097-0142(19940601) 73:11<2779::aid-cncr2820731121>3.0.co;2-u.

8. Uccella S, Morris JM, Bakkum-Gamez JN, Keeney GL, Podratz KC, Mariani A. Bone metastases in endometrial cancer: report on 19 patients and review of the medical literature. Gynecol Oncol. 2013;130(3):474-82. Doi: 
10.1016/j.ygyno.2013.05.010.

9. Madabhavi IV, Patel A, Sarkar MS, Modi MG, Aagre S. Isolated acrometastasis: A rare presenting feature of endometrial carcinoma. J Cancer Res Ther. 2019;15(6): 1402-4. Doi: 10.4103/jcrt.JCRT_394_15.

10. Albareda J, Herrera M, Lopez Salva A, Garcia Donas J, Gonzalez R. Sacral metastasis in a patient with endometrial cancer: case report and review of the literature. Gynecol Oncol. 2008;111(3):583-8. Doi: 10.1016/ j.ygyno.2008.04.005.

11. Myriokefalitaki E, D'Costa D, Smith M, Ahmed AS. Primary bone metastasis as initial presentation of endometrial cancer (stage IVb). Arch Gynecol Obstet. 2013;288(4):739-46. Doi: 10.1007/s00404-013-2956-Z

12. Lane MD, Le HB, Lee S, Young C, Heran MK, Badii M, et al. Combination radiofrequency ablation and cementoplasty for palliative treatment of painful neoplastic bone metastasis: experience with 53 treated lesions in 36 pa- tients. Skeletal Radiol. 2011;40(1):25-32. Doi: 10.1007/ s00256-010-1010-5.

13. Kaya A, Olmezoglu A, Eren CS, Bayol U, Altay T, Karapinar L, et al. Solitary bone metastasis in the tibia as a presenting sign of endometrial adenocarcinoma: a case report and the review of the literature. Clin Exp Metastasis. 2007;24(2):87-92. Doi: 10.1007/s10585-0079061-2.

14. Uharcek P, Mlyncek M, Ravinger J. Endometrial adenocarcinoma presenting with an osseous metastasis. Gynecol Obstet Invest. 2006;61(4):200-2. Doi: 10.1159/ 000091402 .

15. Koukourakis MI, Papadopoulou A, Kyrgias G. Long-term survival of a patient with multiple abdominal metastasis from endometrial carcinoma treated with multi-portal conformal re-irradiation and chemotherapy. Hematol Oncol Stem Cell Ther. 2011;4(1):45-7. Doi: 10.5144/16583876.2011.45. 